\title{
Estado del conocimiento en ecología y conservación de los roquedales de la Argentina: Una revisión
}

\author{
Dulce S. Gómez Carella ${ }^{\bowtie}$; Karina L. Speziale \& Sergio A. Lambertucci \\ Grupo de Investigaciones en Biología de la Conservación, Laboratorio Ecotono, INIBIOMA (CONICET-Universidad Nacional \\ del Comahue). Río Negro, Argentina.
}

\begin{abstract}
RESUMEN. Los roquedales proveen recursos que aprovechan diferentes especies, lo cual los convierte en sitios relevantes para conservar la biodiversidad. Sin embargo, estos sistemas permanecen poco estudiados a nivel global, y la Argentina no es la excepción. Por este motivo, realizamos una revisión bibliográfica para aportar información sobre el estado del conocimiento en relación con el rol ecológico de los roquedales y sobre su importancia como elementos clave de diferentes ecosistemas argentinos. Encontramos 85 artículos que pusieron en evidencia el uso de los roquedales por diferentes especies. De ellos, sólo un tercio se enfocó en el análisis específico de estos sistemas y evaluó, principalmente, su función como hábitat para las especies, seguido por su rol como refugios y, finalmente, como sitios de interacción entre individuos. La literatura científica sugiere que en la Argentina son escasos los trabajos que evalúan de forma apropiada la importancia de los roquedales para la conservación de la biodiversidad, su contribución hacia las personas, el posible impacto antrópico sobre ellos y las acciones que se podrían tomar para favorecer su conservación y el mantenimiento de los servicios que brindan. Esta información es necesaria para diagramar estrategias de manejo adecuadas que incluyan a estos sitios. La falta de dichas estrategias se podría asociar al bajo interés que generan los roquedales como sistemas de estudio, ya que por lo general se los considera como inertes o poco relevantes. Sugerimos incentivar el estudio ecológico de estos ambientes que pueden contener organismos e interacciones novedosas. Además, proponemos acciones específicas para preservar los procesos naturales que allí suceden, y para evaluar y reducir el impacto antrópico con la finalidad de mantener sus funciones.
\end{abstract}

[Palabras clave: biodiversidad, disturbio, hábitat, geodiversidad, servicios ecosistémicos]

AвSTRACt. State of knowledge on the ecology and conservation of rocky outcrops in Argentina: A review. Rocky outcrops provide resources that are used by different species and make them extremely important sites for biodiversity conservation. However, these systems remain poorly studied at a global level, and Argentina is not an exception. For this reason, we reviewed the available literature to provide the state of knowledge about the ecological role of rocky outcrops, and their importance as key landscape elements of Argentine ecosystems. Our review showed 85 articles that evidenced the use of rocky outcrops by different species. Of these, only one third focused on the specific study of these systems, evaluating mainly their function as habitat for species, followed by their role as shelters and, finally, as sites of interaction between individuals. The articles found suggest there are few studies in Argentina which properly evaluate the importance of rocky outcrops for biodiversity conservation, their contribution to people, the possible anthropic impact on them, and the actions that could be taken to favor their conservation and the maintenance of the services they provide. This information is essential for the design of adequate management policies that include these sites. The lack of these policies could be associated to a low interest aroused by those habitats as study systems, generally considered as inert or not very relevant. We suggest encouraging the ecological studies of these environments, which may contain novel organisms and interactions. In addition, we suggest specific actions for the preservation of the natural processes that take place there, and to evaluate and reduce the anthropic impact on them in order to maintain their functions.

[Keywords: biodiversity, ecosystem services, geodiversity, habitat, impact]

\section{INTRODUCCIÓN}

Algunos sistemas naturales ofrecen recursos clave para la supervivencia de diversas especies, y cumplen un papel ecológico cuya importancia puede resultar desproporcionada en relación con su tamaño o disponibilidad (Fitzsimons and Michael 2017; Hunter Jr. et al. 2017). La geodiversidad, definida recientemente como la variedad de materiales, formas y procesos físicos de la superficie terrestre (Hjort et al. 2015), promueve algunos de estos recursos clave, como son las geoformas. Entre la gran variedad de geoformas existentes, los roquedales y los hábitats queéstos generan constituyen sistemas de estudio interesantes por su distribución, sus condiciones microambientales, su amplio rango de tamaños y su grado de aislamiento. Estas características le aportan al paisaje una complejidad que la biota local aprovecha (Porembski and Barthlott 2000). 
Los roquedales se definen como ambientes rocosos que sobresalen por sobre la superficie terrestre (Fitzsimons and Michael 2017) y que constituyen sistemas estables, pero a la vez dinámicos (Hjort et al. 2015). Su estructura genera microhábitats dentro de un mismo afloramiento y, a una escala mayor, le otorga heterogeneidad al paisaje. Esto favorece el mantenimiento y el aumento de la biodiversidad a nivel regional, dado que distintas especies de animales y de plantas pueden usar los roquedales (e.g., Milchunas and Noy-Meir 2002; Michael et al. 2010; Lambertucci and Ruggiero 2013; Speziale and Ezcurra 2015). La heterogeneidad, sumada al aislamiento que caracteriza a estos sistemas, puede resultar en la presencia de una biota especializada y de numerosos endemismos (e.g., Mares 1997; Porembski and Barthlott 2000; Burke et al. 2003; Michael and Lindenmayer 2012).

Los roquedales pueden también presentar condiciones ambientales que fluctúan en el transcurso de los días o de las estaciones (Mares 1997; Larson et al. 2000; Hjort et al. 2015), y que algunas especies pueden aprovechar (Speziale and Ezcurra 2015). Sin embargo, quizás porque resalta su componente inerte, por su área reducida en comparación con otros ambientes o por su aparente resiliencia, el rol que desempeñan estos sistemas suele pasar desapercibido y pocas veces se los tiene en cuenta durante la gestión de planes de conservación. Por lo tanto, los roquedales fueron muy poco estudiados a nivel mundial (Michael et al. 2010; Hunter Jr. et al. 2017). Específicamente, en la Argentina hay poca información al respecto.

En este estudio realizamos una revisión bibliográfica y sintetizamos los trabajos que se llevaron a cabo sobre la ecología de los roquedales en la Argentina. A partir de ellos, aportamos información que respalda el concepto de los roquedales como componentes distintivos de ciertos ecosistemas nativos. A su vez, ponemos de manifiesto la falta de información en relación con el rol que cumplen estos sitios para las especies que los habitan, y la necesidad de generar conocimiento que permita entender la relevancia y función de los roquedales en diversos ambientes de la Argentina. Para mostrar y discutir los trabajos resultantes de dicha revisión organizamos el presente artículo en dos secciones. En la primera describimos los resultados cuantitativos de la búsqueda. En la segunda sección presentamos la clasificación y el resumen de los artículos, y mostramos la información disponible publicada sobre: a) la función de los roquedales como sitios proveedores de hábitat, con las subcategorías de refugio y sitios de interacción; b) los disturbios que impactan los roquedales, y c) la conservación de los roquedales. En esta segunda sección presentamos, en cada caso, un párrafo de introducción general al tema, seguido del resumen de la información encontrada para la Argentina. Finalmente, incluimos una discusión general.

\section{Materiales y MÉTOdos}

Realizamos una revisión sistemática de artículos científicos desarrollados en la Argentina que proporcionaron información sobre la función de los roquedales en los ecosistemas argentinos mediante las plataformas de búsqueda Scopus (www.scopus.com) y Google Académico (https://scholar.google.com.ar). Luego seleccionamos aquellos artículos publicados hasta febrero de 2018, y excluimos los estudios paleontológicos o las investigaciones realizadas en ambientes acuáticos (i.e., roquedales sumergidos). Durante la revisión en Scopus utilizamos palabras clave y la siguiente fórmula de búsqueda, siguiendo la metodología requerida por el buscador: "cliff" OR "outcrop" OR "rock*" OR "inselberg" OR "roquedal" OR "acantilado" OR "pedrero" AND "Argentina". Estos términos se emplearon para la búsqueda en Google Académico, y se complementaron con los términos "rocky outcrop" y "biodiversity" ; éstos permitieron obtener un mayor número de resultados en esta plataforma. Se revisaron todos los artículos encontrados en Scopus y los primeros 150 de Google Académico. También exploramos la bibliografía citada en los artículos encontrados en la búsqueda. Descartamos términos ambiguos como "roqueríos", "áreas rocosas", "barrancos" o "pendientes", que en su gran mayoría no se consideran roquedales según la definición que propusieron Fitzsimons y Michael (2017), sino zonas con piedras sueltas, pendientes o derrumbes no rocosos. Excluimos los artículos donde citaban la palabra "roquedales", que carecieron de claridad y difícilmente proporcionaron el uso específico de las especies en estos ambientes (i.e., que sólo nombraran una especie asociada a un roquedal). De acuerdo con los resultados de cada investigación clasificamos los artículos seleccionados en: a) roquedales como hábitat 
(i.e., sitios donde los organismos pueden desarrollar su ciclo de vida); dentro de esta categoría distinguimos sitios usados para refugio y sitios de interacción social para la biota estudiada; b) disturbios sobre roquedales, y c) conservación de la biodiversidad y servicios provistos por los roquedales. Analizamos en detalle las dos subcategorías dentro de "roquedales como proveedores de hábitat", dado que fueron resaltadas en particular por la bibliografía. Cada artículo puede estar asignado a más de una categoría, de modo que los resultados en las categorías previas no corresponden con el número total de trabajos.

Además, de cada estudio obtuvimos la información disponible respecto al año de la publicación, al tipo de ecorregión (e.g., Bosques patagónicos, Espinal, Monte de Sierras y Bolsones), la altura de su vegetación (baja, media y alta), la estructura utilizada del roquedal (e.g., grietas, cuevas, acantilados, repisas), el grupo taxonómico y la especie estudiada. Aspectos como la altura de la vegetación pueden ser importantes para esta investigación porque es probable que la función de un roquedal varíe según la complejidad del paisaje donde se encuentre.
Finalmente, en aquellos trabajos en los que se incluyeron los nombres científicos de las especies estudiadas se consideró el estatus de las mismas (IUCN 2018). Al mismo tiempo, y en caso de estar incluida en el trabajo, se registró la importancia ecológica, económica y cultural de las especies o los roquedales.

\section{Resultados}

Encontramos 85 artículos realizados sobre especies que se vinculan de alguna forma a roquedales en la Argentina. El 71\% de esos trabajos (60 artículos) menciona a los roquedales como parte del estudio, sin ser el análisis de estos sitios su principal objetivo. Del 29\% restante (25 artículos), 80\% (20 artículos) evaluó de manera específica la función de los roquedales como hábitat para distintas especies, 60\% (15 artículos) analizó dichos sistemas por la protección que brindan, y $24 \%$ ( 6 artículos) se enfocó en su importancia como sitios de encuentro entre individuos y espacios donde ocurren intercambios de información.

El primer trabajo realizado sobre aspectos ecológicos de los roquedales en la Argentina

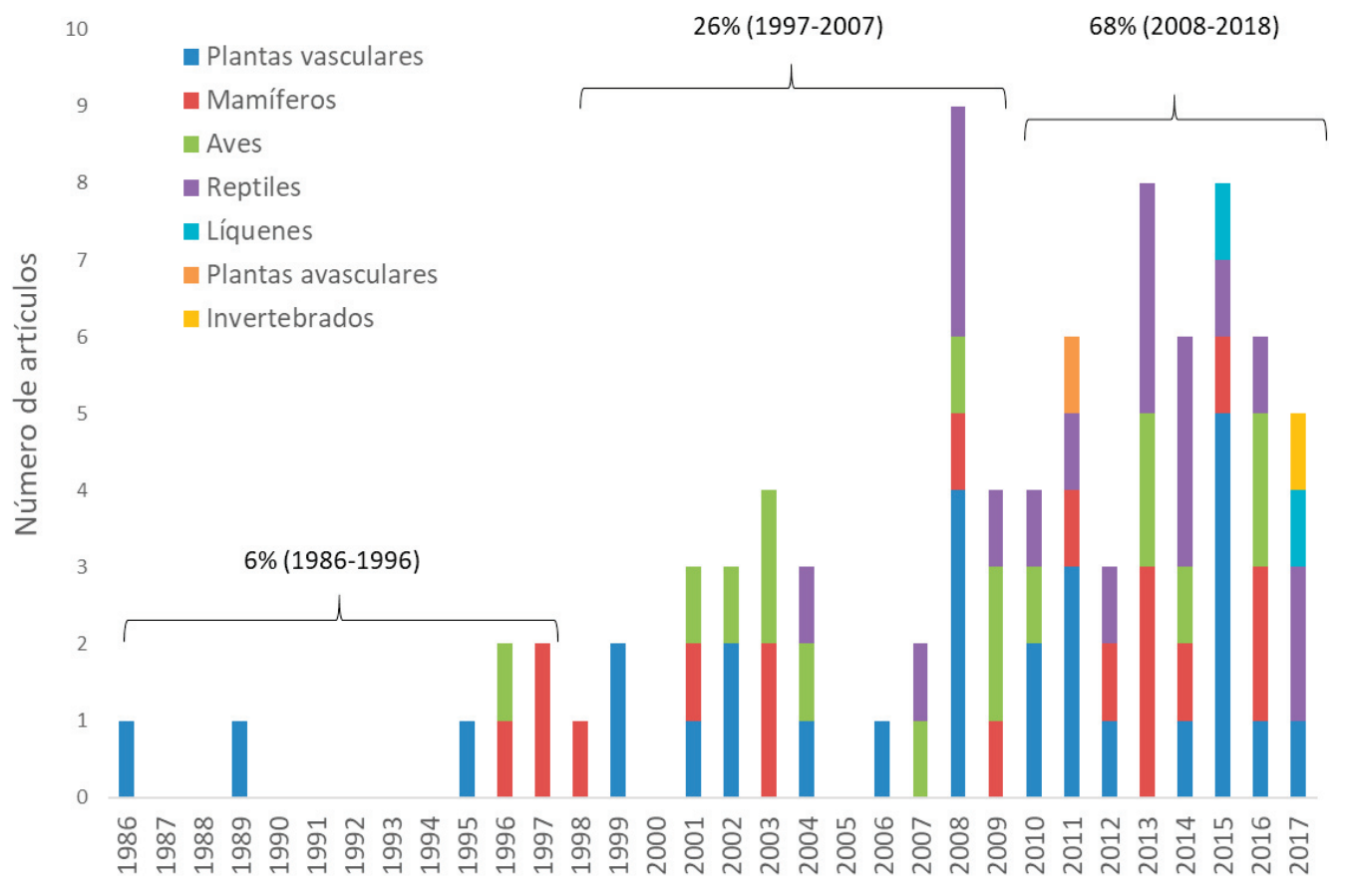

Año de estudio

Figura 1. Número de artículos publicados que estudiaron plantas vasculares, mamíferos, aves, reptiles, líquenes, plantas celulares e invertebrados en roquedales entre 1986 y 2017 en la Argentina.

Figure 1. Number of published articles studying vascular plants, mammals, birds, reptiles, lichens, cellular plants and invertebrates in rocky outcrops between 1986 y 2017 in Argentina. 
fue publicado en 1986. A partir de este año y hasta 1996, el porcentaje de investigaciones fue bajo (6\% de los trabajos). Durante el periodo 1997-2007, este porcentaje se elevó al 26\%. Finalmente, más de la mitad de los estudios en el tema se llevó a cabo en los últimos 10 años (68\%, período 2008-2018) (Figura 1). Las plantas vasculares fueron el primer taxón en ser estudiado en relación a los roquedales, seguidas por los mamíferos y las aves, que comenzaron a ser objeto de estudio a partir de 1996. Los invertebrados corresponden al último grupo en ser incorporado a este tipo de estudios, a partir del año 2017 (Figura 1).

Con relación a la altura de la vegetación, 58\% de los trabajos (49 artículos) se realizó en hábitats caracterizados por vegetación baja, con poca estructura horizontal, 28\% (24 artículos) corresponde a hábitats de vegetación alta, y 13\% (11 artículos) pertenece a ambientes de vegetación intermedia (Tabla 1, Figura 2). En el $48 \%$ de los trabajos (41 artículos) se estudiaron los roquedales en general, sin especificar el tipo de ambiente que usan las especies. En los estudios restantes (44 artículos), 45\% de los trabajos se enfocó en el uso de grietas, $34 \%$ el de las paredes acantiladas y $21 \%$ se orientó hacia otro tipo de ambientes como promontorios, repisas, cuevas y depresiones (Anexo 1).
A nivel de grupo taxonómico, de las especies de plantas vasculares estudiadas, la mayoría pertenece a la familia Asteraceae (géneros Dolichlasium, Eupatorium, Artemisia, Grindelia y Baccharis), seguidas por las familias Fabaceae (géneros Cercidium y Apurimacia), Poaceae (género Nassella), Rosaceae (géneros Polylepis y Prunus), Solanaceae (géneros Solanum y Fabiana) y Cupressaceae (género Austrocedrus). El 16\% restante corresponde a otras familias menos representadas (Tabla 2 ).

La mayoría de los mamíferos estudiados pertenece al orden de los roedores; el género Lagidium fue el más estudiado, y lo siguieron en importancia Abrocoma y Octomys. En cuanto a las aves, se estudiaron seis órdenes, cada uno representado por un sólo género. Los más investigados fueron los Catártidos (género Vultur) y los Suliformes (género Phalacrocorax). Todas las especies de reptiles estudiadas pertenecen al orden Squamata, y el género más representado es Phymaturus (Tabla 3; Anexo 1).

Los taxa menos estudiados fueron los líquenes, los invertebrados, los protistas y las plantas celulares. Sólo tres de los 85 trabajos $(<4 \%)$ estudiaron líquenes, y dos de ellos a especies pertenecientes a las familias Teloschistaceae y Parmeliaceae.

Tabla 1. Porcentaje (\%) y número $(\mathrm{N})$ de trabajos realizados en roquedales localizados en diferentes ecorregiones. Las ecorregiones se agruparon en base a la altura de la vegetación (baja, media y alta). Ver más detalles de las ecorregiones correspondientes en la Figura 2.

Table 1. Percentage (\%) and number of articles (N) found that studied rocky outcrops from different ecoregions. We grouped the ecoregions based on the height of the vegetation: low (baja), medium (media), high (alta). For more details on the corresponding ecoregions, see Figure 2.

\begin{tabular}{lclclc}
\hline \multicolumn{1}{c}{ Baja } & $\mathrm{N}(\%)$ & \multicolumn{1}{c}{ Media } & $\mathrm{N}(\%)$ & \multicolumn{1}{c}{ Alta } & $\mathrm{N}(\%)$ \\
\hline Estepa Patagónica & $43.52(37)$ & Espinal & $7.05(6)$ & Bosques patagónicos & $10.58(9)$ \\
Pampa & $8.23(7)$ & Monte de sierras y bolsones & $7.05(6)$ & Selva paranaense & $1.17(1)$ \\
Puna & $1.17(1)$ & Monte de llanuras y mesetas & $4.70(4)$ & Campos y malezales & $1.17(1)$ \\
Altos Andes & $4.70(4)$ & Chaco seco & $9.41(8)$ & & $12.94(12)$ \\
\cline { 3 - 6 } & & & $28.23(24)$ & &
\end{tabular}

Tabla 2. Porcentaje (\%) y número (N) de artículos que estudiaron diferentes familias de plantas vasculares de las Clases Gymnospermae, Monocotyledoneae y Magnoliposida en roquedales de la Argentina.

Table 2. Percentage (\%) and number of articles (N) that studied different families of vascular plants of the classes Gymnospermae, Monocotyledoneae and Magnoliposida in rocky outcrops of Argentina.

\begin{tabular}{|c|c|c|c|c|c|}
\hline \multicolumn{2}{|c|}{ Gymnospermae } & \multicolumn{2}{|c|}{ Monocotyledoneae } & \multicolumn{2}{|c|}{ Magnoliopsida } \\
\hline Orden & Familia & Orden & Familia & Orden & Familia \\
\hline \multirow[t]{6}{*}{ Cupressales } & Cupressaceae $2(8 \%)$ & Poales & Poaceae $3(12 \%)$ & Aquifoliales & Aquifoliaceae $1(4 \%)$ \\
\hline & & Zingiberales & Zingiberaceae $1(4 \%)$ & Asterales & Asteraceae 7 (28\%) \\
\hline & & & & Fabales & Fabaceae 4 (16\%) \\
\hline & & & & Lamiales & Lamiaceae 1 (4\%) \\
\hline & & & & Rosales & Rosaceae 3 (12\%) \\
\hline & & & & Solanales & Solanaceae $2(8 \%)$ \\
\hline
\end{tabular}




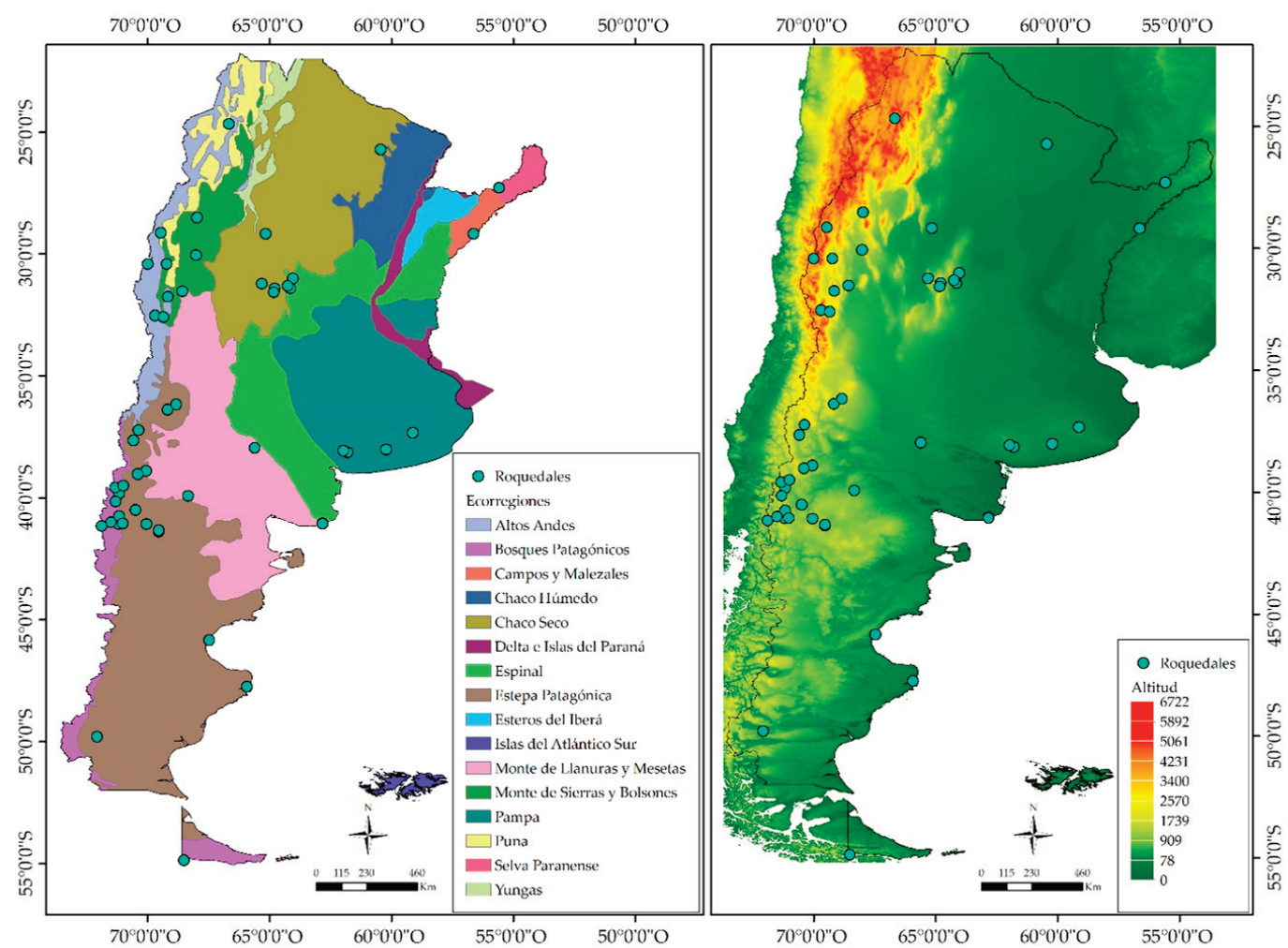

Figura 2. Mapa de elevación (izquierda) y ecorregiones (derecha). Localización de los roquedales estudiados en los artículos analizados (puntos celestes).

Figure 2. Elevation (left) and ecoregions map (right). Location of the rocky outcrops studied in the analyzed articles (blue dots).

Otras tres investigaciones se enfocaron en arañas migalomorfas de la familia Migidae, protistas de la clase Myxomycetes y algas y plantas celulares de diversos grupos. La información extraída de cada artículo se encuentra detallada en el Anexo I.

\section{Roquedales como hábitat}

Uno de los rasgos principales que caracteriza a los roquedales como buenos hábitats es su heterogeneidad; en particular, el gradiente de condiciones ambientales que ofrecen y que no se encuentra en la matriz circundante (Michael et al. 2010). Su topografía compleja resulta de la formación de estructuras como cuevas, grietas y piletas temporales, rasgos que, según su composición geológica, incrementan la diversidad del hábitat y el impacto de los factores erosivos dominantes (Burnett et al. 1998; Larson et al. 2000; Hjort et al. 2015) (Figura 3). En un ambiente donde las condiciones son fluctuantes, los roquedales pueden representar sitios estables que otorgan seguridad espacial y temporal (Tscharntke et al. 2005). En cambio, en otras ocasiones, su dinamismo ofrece un hábitat particular que pueden aprovechar diferentes especies.
La estructura de los roquedales genera microclimas que presentan ciclos periódicos, lo que permite a los animales elegir qué parte del roquedal utilizar en diferentes momentos del día (Larson et al. 2000).

De los 43 artículos que investigaron el rol de los roquedales como proveedores de hábitat en la Argentina, 15 estudiaron mamíferos, 14 plantas vasculares, 9 aves, 3 reptiles y 1 líquenes. Los trabajos sobre mamíferos se enfocaron principalmente en roedores, dado que las cuevas y las grietas conforman microhábitats ideales para la construcción de madrigueras y la obtención de beneficios térmicos (Mares 1997; Sobrero et al. 2010; Campos et al. 2013; Andino et al. 2016). Algunas de estas especies, que se mueven con dificultad sobre tierra, pueden desplazarse con facilidad y rapidez sobre sustratos rocosos gracias a que poseen adaptaciones morfológicas especiales (Mares and Lacher 1987; Galende and Trejo 2003). Tanto el chinchillón (Lagidium viscacia) como las chinchillas del género Abrocoma presentan características que les otorgan agilidad, como uñas reducidas y almohadillas plantares especiales para la tracción y amortiguación de impactos (Braun and Mares 1996; Walker et al. 
Tabla 3. Porcentaje (\%) y número de artículos $(\mathrm{N})$ que estudiaron diferentes órdenes y géneros de mamíferos, aves y reptiles en roquedales de la Argentina. El porcentaje de cada grupo taxonómico está calculado en base al total de artículos sobre roquedales a nivel de orden, de acuerdo con el número de artículos por grupo taxonómico, y a nivel de género, considerando el número de artículos por orden.

Table 3. Percentage (\%) and number of articles $(\mathrm{N})$ that studied different orders and genera of mammals, birds and reptiles in rocky outcrops of Argentina. The percentage of each taxonomic group is calculated based on the total number of articles on rocky outcrops, the percentage of each order is calculated based on the number of articles per taxonomic group, and the percentage of each genus is calculated based on the number of articles per order.

\begin{tabular}{|c|c|c|c|c|c|}
\hline \multicolumn{2}{|c|}{ Mamíferos 22.35\% (19) } & \multicolumn{2}{|c|}{ Aves $16.47 \%$ (14) } & \multicolumn{2}{|c|}{ Reptiles 22.35\% (19) } \\
\hline Orden & Género & Orden & Género & Orden & Género \\
\hline \multirow{5}{*}{$\begin{array}{l}\text { Rodentia } \\
84.21 \%(16)\end{array}$} & Lagidium $47.37 \%$ (9) & Catartiformes $42.86 \%(6)$ & Vultur $42.86 \%(6)$ & \multirow{5}{*}{$\begin{array}{l}\text { Squamata } \\
100 \%(19)\end{array}$} & Phymaturus $89.47 \%$ (17) \\
\hline & Abrocoma $15.79 \%$ (3) & Suliformes $21.43 \%$ (3) & Phalacrocorax $21.43 \%$ (3) & & Liolaemus $10.53 \%$ (2) \\
\hline & Octomys $15.79 \%$ (3) & Psitaciformes $14.29 \%$ (2) & Cyanoliseus $14.29 \%$ (2) & & \\
\hline & Microcavia 5.26\% (1) & Estrigiformes 7.14\% (1) & Bubo $7.14 \%$ (1) & & \\
\hline & & Pelecaniformes 7,14\% (1) & Theristicus $7.14 \%$ (1) & & \\
\hline \multirow{3}{*}{$\begin{array}{l}\text { Carnívora } \\
15.79 \% \text { (3) }\end{array}$} & Conepatus $5.26 \%(1)$ & Accipitriformes 7,14\% (1) & Geranoaetus 7.14\% (1) & & \\
\hline & Pseudalopex $5.26 \%$ (1) & & & & \\
\hline & Puma $5.26 \%(1)$ & & & & \\
\hline
\end{tabular}

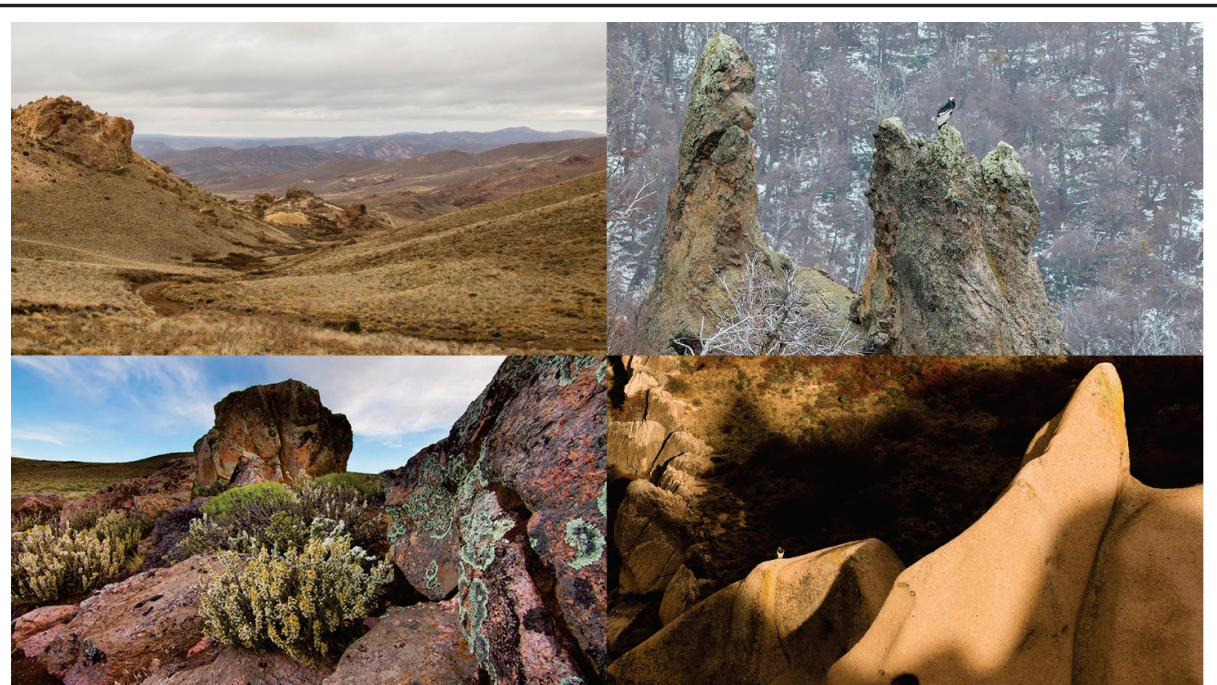

Figura 3. Ejemplos de roquedales y sus estructuras usadas por diferentes especies saxícolas (fotos: Gonzalo Ignazi).

Figure 3. Examples of rocky outcrops and their structures used by different saxicolous species (photos: Gonzalo Ignazi).

2003; Galende and Raffaele 2008; Taraborelli et al. 2011).

Numerosas especies de aves aprovechan los acantilados y los paredones que forman los roquedales para nidificar o descansar en sus repisas y grietas (Punta et al. 2003; Rosciano et al. 2013; Roesler et al. 2014; Fandiño et al. 2017). El cóndor andino (Vultur gryphus) es una especie que usa este recurso al descansar en dormideroscomunales formados por repisasen acantilados (Lambertucci et al. 2008) y también al nidificar en cuevas o repisas (Lambertucci and Mastrantuoni 2008). Se estima que este tipo de agrupamiento les permitiría obtener beneficios termorregulatorios (Lambertucci and Ruggiero 2013). En el caso de los reptiles, se le dedicó una atención especial al estudio de los géneros Phymaturus (e.g., Cabezas-Cartes et al. 2010; Debandi et al. 2012; Castro et al. 2013; Corbalán and Debandi 2013) y Liolaemus (Avila et al. 2012), especialmente en la Patagonia. Su presencia en roquedales es habitual debido a que aprovechan sus grietas para refugiarse y la exposición solar y la acumulación de calor en la superficie para regular su temperatura corporal (Corbalán and Debandi 2013). Se considera que estos géneros son importantes por su estado de conservación vulnerable (Quinteros et al. 2012; Cabezas-Cartes et al. 2014) y porque muchas especies representan endemismos (Córdoba et al. 2015).

Casi todos los estudios sobre la vegetación asociada a roquedales en la Argentina se realizaron en base a plantas vasculares $(28$ de 29 artículos). Las especies vegetales que pueden vivir en suelos poco profundos 
tienen la capacidad de crecer en sustratos rocosos gracias a ciertas ventajas asociadas a estos ambientes. Ejemplos de ellas son la acumulación de materia orgánica en grietas (que facilita el almacenamiento de humedad [Porembski and Barthlott 2000; Gandullo and Faggi 2006; Kristensen and Frangi 2015]) y la heterogeneidad climática entre diferentes caras de un mismo roquedal (lo que permitiría la presencia de floras de ambientes fríos y templados en el mismo afloramiento [Speziale and Ezcurra 2015]). Estas características de los roquedales, junto con otras definidas tanto por su formación geológica como por su grado de exposición solar, determinarían en gran parte las diferencias que pueden encontrarse entre comunidades de distintos afloramientos (Speziale and Ezcurra 2012; Kristensen and Frangi 2015). En la Patagonia se estudiaron especies saxícolas, especialmente en algunos bosques y en el ecotono bosque-estepa (Anchorena and Cingolani 2002; Oddi et al. 2010; Landesmann et al. 2015). Además, se realizaron trabajos en las provincias de Córdoba (Menghi et al. 1989; Vischi et al. 2004), en los llanos del noroeste de San Juan (Carretero et al. 2010) y en la Región Pampeana (Kristensen and Frangi 2015; Herrera et al. 2017), principalmente enfocados en el estudio de la composición de las comunidades que ocupan esos sitios.

\section{Sitios usados como refugio}

Las plantas y los animales se enfrentan a diario con situaciones que pueden representar una amenaza a su supervivencia y que son de origen tanto natural como antrópico. Entre éstas, los incendios, la fluctuación climática y los depredadores son algunas de las amenazas más habituales (e.g., Walker et al. 2000; Lambertucci and Ruggiero 2013; Landesmann et al. 2015). Una de las maneras de evitar sus impactos es mediante la utilización de refugios. Gracias a la presencia de estructuras como repisas, grietas y cuevas, los roquedales pueden ser utilizados como resguardo frente a condiciones adversas o peligrosas por distintas especies (Mares 1997; Larson et al. 2000; Walker et al. 2000; Milchunas and NoyMeir 2002).

De los 27 trabajos que estudiaron el rol de los roquedales como proveedores de refugio en la Argentina, 12 se enfocaron en mamíferos (e.g., Microcavia australis, L. viscacia, Abrocoma schistacea), 8 en plantas vasculares (e.g., Fabiana imbricata, Austrocedrus chilensis, Polylepis australis), 5 en aves (Phalacrocorax atriceps,
$P$. magellanicus y $V$. gryphus) y 2 en reptiles (Phymaturus spectabilis y P. tenebrosus). Estos trabajos estudiaron cómo la formación de galerías y de espacios inaccesibles, ya sea por su altura o profundidad, son aprovechados por numerosas especies de animales y plantas (e.g., Lambertucci and Ruggiero 2013; Landesmann et al. 2015). Las repisas, las pendientes pronunciadas y las grietas estrechas son ejemplos de buenos refugios que usan los reptiles, las aves y los roedores (Galende and Grigera 1998; Walker et al. 2000; Campos et al. 2013; Cabezas-Cartes et al. 2014).

Muchas especies usan los roquedales para protegerse de depredadores. Por ejemplo, trabajos realizados sobre el cóndor andino y el cormorán de las rocas (P. magellanicus) sugieren que cuando estas especies usan las repisas para pernoctar y nidificar se reduciría el riesgo de depredación tanto terrestre como aérea (Punta et al. 2003; Lambertucci and Ruggiero 2013). En ocasiones, este recurso adquiere tal importancia que los animales pueden modificar sus hábitos para permanecer en ese hábitat; así priorizan el resguardo a la obtención de comida (Walker et al. 2000, 2003). Un ejemplo de este comportamiento sería el del chinchillón patagónico, que adaptaría su dieta para poder forrajear en la vecindad de las madrigueras sin tener que desplazarse distancias que aumenten el riesgo de depredación (Galende and Raffaele 2012). Las especies vegetales también utilizan los afloramientos como refugio; incluso, podrían persistir en los roquedales cuando ya han desaparecido de la matriz circundante debido a la presión por pastoreo (Milchunas and NoyMeir 2002; Speziale and Ezcurra 2012).

Cuando el clima de una región cambia de forma drástica en poco tiempo o alcanza intensidades perjudiciales en temperatura, precipitaciones o vientos, la estabilidad que proveen los microhábitats de los roquedales se convierte en un refugio fundamental para algunas especies (Cabezas-Cartes et al. 2014). Las caras de la roca que se encuentran más expuestas a la luz solar y en dirección contraria al viento suelen ser las que distintas especies de aves y mamíferos eligen para establecer sus nidos, sitios de descanso y madrigueras (Punta et al. 2003; Lambertucci and Ruggiero 2013; Taraborelli et al. 2015; Andino et al. 2016). Los espacios sombríos y frescos del roquedal también pueden ser aprovechados, especialmente por especies vegetales que presentan poca tolerancia a 
las altas temperaturas (Speziale and Ezcurra 2015).

En varias regiones de la Argentina, la combinación deunclima secocon temperaturas elevadas y acumulación de biomasa vegetal resulta en la ocurrencia frecuente de incendios (Kitzberger et al. 1997). Si bien el fuego es fundamental para determinar la estructura de algunas comunidades vegetales (Morrison et al. 1995; Dudinszky and Ghermandi 2013), existen otras que, al igual que muchos animales, resultan perjudicadas por el fuego. Para estas especies, los roquedales pueden constituir un refugio muy valioso, dado que, a diferencia de lo que ocurre en la matriz circundante, el fuego no suele afectarlos (Cantero et al. 2017). Estudios realizados en Córdoba y en la Patagonia argentina sugieren que ciertas especies vegetales (e.g., F. imbricata, $A$. chilensis y $P$. australis) pueden crecer en los afloramientos rocosos y en sus cercanías gracias a que éstos les brindan protección contra incendios (Renison et al. 2002; Oddi et al. 2010; Souto et al. 2012; Landesmann et al. 2015).

\section{Sitios de interacción social}

Los animales que presentan algún tipo de comportamiento social pueden utilizar los roquedales y beneficiarse de su estructura. Algunas de las actividades que suelen llevar a cabo en estos sitios son la vigilancia, la defensa de territorio y la formación de grupos familiares, entre otras (Mares and Lacher 1987; Mares 1997). En estos sitios también pueden realizarse intercambios de información entre individuos o grupos de individuos (Ward and Zahavi 1973; Mares 1997).

Para la Argentina, encontramos 11 trabajos ( 5 en mamíferos y 6 en aves) que estudiaron las interacciones bióticas que tienen lugar en roquedales. Estas fueron principalmente entre individuos de una misma especie (Taraborelli and Moreno 2009; Taraborelli 2011), aunque también existen entre especies (Donázar and Feijóo 2002). Algunas ocurren en madrigueras que generalmente se encuentran en grietas profundas, donde un comportamiento social como la reproducción grupal resulta en la obtención de beneficios térmicos (Taraborelli and Moreno 2009). La estructura externa del roquedal también es de mucha utilidad para algunas especies de roedores que conforman grupos familiares, como ocurre con el chinchillón patagónico. En este caso, las rocas son usadas como postas de vigilancia debido a que los individuos tienen la facilidad de dar alarma ante la presencia de posibles depredadores (Mares 1997; Galende and Raffaele 2008). La altura del roquedal también favorece a las especies que demarcan su territorio mediante comunicación química, como las chinchillas del género Abrocoma, que establecen letrinas masivas sobre pilas de rocas (Taraborelli et al. 2011). Es posible que este comportamiento se deba a la mayor dispersión que alcanza el olor de las defecaciones realizadas en las zonas altas (Mares 1997). En cuanto a las aves, se cree que al usar dormideros comunales las especies rapaces podrían realizar intercambios de información en los acantilados donde perchan e interactúan de manera jerárquica (Donázar et al. 1996; Donázar and Feijóo 2002).

\section{Disturbios sobre roquedales}

La superficie inerte de la roca suele transmitir una imagen general de resiliencia de estos ambientes (Bauer et al. 2017) y de las especies que los habitan, lo que muchas veces lleva a subestimar las amenazas a las cuales pueden estar expuestos. Los impactos negativos que ocurren sobre los roquedales son, sobre todo, de origen antrópico, y suelen ocurrir como consecuencia secundaria de prácticas con fines económicos, como, por ejemplo, la minería o el sobrepastoreo del ganado (Clements et al. 2006; Michael et al. 2010; Hjort et al. 2015; Fitzsimons and Michael 2017). En los últimos años también tomó relevancia el daño que causan las actividades turísticas y deportivas, como en el caso de la escalada. Este deporte posibilita el acceso de personas a lugares remotos a través de rutas nuevas en ambientes naturales, muchas veces sin ningún tipo de control (Holzschuh 2016).

En la Argentina se realizaron sólo tres estudios que describen o evalúan impactos puntuales de las actividades antrópicas sobre algunas especies en particular; pero estos trabajos no consideran los daños que esas actividades ocasionan sobre la comunidad que habita los roquedales. Por ejemplo, en la Patagonia argentina se observaron disturbios en nidos de cóndor andino por parte del público. En ocasiones, esta especie nidifica en rocas accesibles para turistas y escaladores, lo cual puede llevar a que los huevos sean eventualmente abandonados o a que los individuos se acostumbren a la presencia de los seres humanos (Lambertucci and Speziale 2009). También se llevaron a cabo trabajos sobre la flora saxícola en el 
centro del país, en particular en zonas donde los afloramientos son muy aprovechados para la explotación minera y, en consecuencia, se están convirtiendo en sitios vulnerables, con demandas crecientes proyectadas a futuro (Cantero et al. 2014, 2017). Sin embargo, esta es una temática en la que hacen falta estudios con evaluaciones más generales en cuanto a los impactos que sufren las comunidades habitantes de los roquedales en distintas zonas del país y bajo diferentes impactos antrópicos.

\section{Conservación de la biodiversidad y servicios que proveen los roquedales}

Si bien el rol que cumplen los roquedales como hábitats, refugios y sitios de interacción es fundamental para mantener y proteger la biodiversidad, su importancia también se refleja en la contribución que brindan a los seres humanos. Esta contribución puede ser clave para el desarrollo económico y cultural de una región como ocurre, por ejemplo, con los beneficios asociados al atractivo del paisaje (Moncrieff 2000; Clements et al. 2006; Rossi and Webb 2007; Lewis and Lewis 2017). Así, el valor ecológico, cultural y económico de los roquedales los convierten en sitios que deben ser considerados al momento de establecer e implementar pautas de manejo y conservación, $\mathrm{e}$, incluso, al crear reservas.

Desde el punto de vista de la conservación de la biodiversidad en la Argentina encontramos 19 trabajos que mostraron el uso de los roquedales por parte de especies consideradas casi amenazadas, en peligro de extinción, o de preocupación menor, pero con su población en descenso (e.g., Frere and Gandini 2001; Corbalán and Debandi 2013; Lambertucci and Ruggiero 2016), que representan endemismos (e.g., Puig et al. 1998; Funes and Cabido 2008; Galende and Raffaele 2008; Herrera et al. 2017) o que son especies nuevas para la ciencia (e.g., Abdala et al. 2008; Scolaro and Ibargüengoytía 2008; Søchting et al. 2014; Ciciarelli 2015). Por este motivo, las prácticas de conservación deberían enfocarse en la preservación de las características que permiten a los roquedales cumplir funciones importantes para la supervivencia de estas especies que dependen de ellos. Por ejemplo, su rol como conectores entre ambientes favorables para una flora sensible a la temperatura (Speziale and Ezcurra 2015) y como hábitats que ofrecen refugio para muchos individuos y que albergan una gran diversidad específica (Lambertucci et al. 2008; Pia et al. 2013; Rosciano et al. 2013; Lambertucci and Ruggiero 2016).
En ocasiones se extraen especies de los roquedales para utilizarlas con fines económicos, como ocurre con plantas de uso medicinal (Cantero et al. 2017) o comercial. Por ejemplo, especies del género Grindelia se pueden usar para fabricar productos como pintura, goma y papel, mientras que Ilex affinis suele utilizarse como sustituto de la yerba mate (Roitman 1999; Keller and Giberti 2011). En el caso de productos de origen animal, sólo se reconoció la explotación de colonias de cormorán imperial (P. atriceps) para la obtención de guano (Punta et al. 2003). Además, uno de los trabajos encontrados remarca la importancia de los roquedales como sitios arqueológicos (Tassara and Cenizo 2014) y resalta la necesidad de implementar planes de conservación para protegerlos.

\section{DiSCUSIÓN GENERAL}

En la Argentina, el estudio de los roquedales creció en el último tiempo, centrándose principalmente en plantas, mamíferos, reptiles y aves que aprovechan los afloramientos como hábitats, refugios y sitios de interacción social. El incremento en el número de trabajos publicados, en especial en la última década, podría sugerir que existe un mayor interés en los roquedales como sistemas de estudio. Sin embargo, los trabajos específicos en esta y otras temáticas (e.g., invasiones biológicas) aún son escasos en relación a lo publicado en otros países (Speziale et al. 2012; Fitzsimons and Michael 2017), y es probable que el incremento temporal sólo refleje el aumento general en la cantidad de publicaciones realizadas en el país. Algunos de los estudios encontrados para la Argentina demuestran que los roquedales pueden albergar especies distintas de las que se encuentran en la matriz circundante (Speziale and Ezcurra 2012; Lambertucci and Ruggiero 2016), lo cual los transforma en elementos especiales del paisaje capaces de contener endemismos y una riqueza de especies particular. Esto concuerda con estudios realizados en otros países, que demuestran la importancia de los afloramientos rocosos como islas de biodiversidad (Porembski et al. 1997; Clements et al. 2006); no obstante, aún faltan estudios comparativos que exploren este aspecto para los roquedales de la Argentina. Esta falta se pone de manifiesto al observar la distribución limitada de los trabajos encontrados (Figura 2), aun cuando en la Argentina existen otros sistemas montañosos y sitios donde muy probablemente se encuentren roquedales de gran importancia para diversas especies. 
Trabajos realizados en diferentes partes del mundo estudian especies que desarrollan adaptaciones particulares para aprovechar estos hábitats, o que por sus características biológicas pueden utilizar rasgos inusuales de los mismos. Algunos ejemplos son los líquenes y las plantas metalófitas que crecen sobre rocas de serpentina (Paukov 2009), y que por su capacidad de acumular metales pesados pueden existir en superficies poco aptas para la mayoría de las especies. Los anfibios también usan los roquedales, e, incluso, pueden beneficiarse de la naturaleza efímera de las piletas temporales que se forman en sus depresiones (Köhler and Böhme 1996). Otro caso interesante es el de los insectos sociales, que pueden desplegar conductas inusuales como el establecimiento de nidos limitantes para adaptarse a la vida en roquedales y evitar la competencia cuando los sitios donde nidificar son escasos (Czechowski 2004). Sin embargo, en la Argentina se llevaron a cabo pocos estudios para evaluar si las especies poseen adaptaciones particulares a la vida en roquedales, por lo cual ampliar su estudio podría resultar de gran relevancia para conocer mejor la dinámica de especies saxícolas locales.

Otro punto fundamental a explorar y que fue escasamente considerado en la Argentina es el de los disturbios antrópicos más frecuentes que afectan a los roquedales. El daño sobre ellos suele comenzar en la matriz circundante, donde puede ocurrir, por ejempo, la compactación del suelo. Este efecto promueve la erosión y perjudica a las especies vegetales y su fauna asociada (Cole 1993); además, generan un espacio que puede ser aprovechado por especies invasoras que se dispersan rápidamente (Fitzsimons and Michael 2017). Las causas más comunes de daño son la circulación de ganado, vehículos y seres humanos, así como también el impacto que generan las actividades recreacionales y los coleccionistas de reptiles (Pike et al. 2010). El ganado usa los roquedales para pasar la noche, lo cual puede transformarlos en sitios inadecuados como hábitat no sólo por el pisoteo, sino también por el pastoreo, muchas veces selectivo (Burke et al. 2003), y por la acumulación de materia fecal (Michael et al. 2010). Por otra parte, la creación de canteras y la actividad minera en general constituyen una amenaza a mayor escala (Hjort et al. 2015) que tampoco se estudió en la Argentina. También es común que los roquedales escénicamente bellos o culturalmente importantes reciban visitas constantes de turistas que pueden afectar su estado de conservación. Trabajos realizados en las cuevas de Wave Rock, en Australia, demostraron cómo el flujo constante de turistas modificó el suelo por erosión (Rossi and Webb 2007). El acceso descontrolado y continuo de gente puede dar lugar a otros tipos de disturbios accidentales o intencionados, como la contaminación del espacio y sus cuerpos de agua, los graffitis (Moncrieff 2000; Hjort et al. 2015; Lewis and Lewis 2017) y la perturbación de la vida salvaje (Cole 1993). También la modificación de la arquitectura de los roquedales por remoción y traslado de rocas presenta consecuencias a largo plazo para las comunidades que viven en ellos, dado que los cambios más pequeños pueden causar la fragmentación del hábitat y la pérdida de conexiones que resultan esenciales para las especies (Michael et al. 2010; Hjort et al. 2015; Fitzsimons and Michael 2017).

Sumado a su importancia intrínseca y ecológica, los roquedales poseen valor utilitario y pueden proveer servicios ecosistémicos cuya valoración económica, incluso, se cuantificó en otros países del mundo. La Contribución de la Naturaleza a la Gente (NCP por sus siglas en inglés o CNG por su traducción al castellano) (Díaz et al. 2018) puede ser directa, como es el caso de los afloramientos que representan fuentes de agua para irrigación y consumo humano, o indirecta, como ocurre con las cuevas que albergan especies de murciélagos polinizadores y dispersores de semillas (Clements et al. 2006). Otra CNG económicamente importante es el geoturismo, impulsado por el valor estético (Burnett et al. 1998; Clements et al. 2006; Hjort et al. 2015), cultural y religioso que los roquedales presentan en muchos países (Clements et al. 2006; Hjort et al. 2015). Algunas cuevas y afloramientos conservan valores asociados a la historia de diversos pueblos originarios (Rossi and Webb 2007). Un ejemplo de esto es la Cueva de las Manos, en la provincia de Santa Cruz. Si bien el turismo sin control puede generar impactos negativos (Rossi and Webb 2007), es importante resaltar que el turismo organizado puede ser una estrategia de conservación clave (Brightsmith et al. 2008). En la Argentina es necesario llevar adelante estudios que resalten el valor intrínseco de los roquedales y estimen el rol que cumplen para la biodiversidad y la CNG en sus diferentes niveles. Estos estudios lograrían valorizar su importancia para el mantenimiento de la biodiversidad y para la provisión de servicios 
útiles para los seres humanos, y permitirían el diseño de estrategias de conservación adecuadas.

\section{CONSIDERACIONES FINALES}

Los problemas relacionados con la conservación de los roquedales pueden deberse a la falta de conocimiento ecológico básico sobre estos ambientes en la Argentina y al desconocimiento de las autoridades, del ámbito científico y del público general sobre sus funciones e importancia (Larson et al. 2000; Fitzsimons and Michael 2017; Hunter Jr. et al. 2017). Es posible que la falta de una definición clara de estos sistemas perjudique su estudio o comprensión. Por ejemplo, algunos trabajos sobre especies que usan los roquedales pueden haber quedado fuera de nuestra búsqueda por el uso de criterios o terminologías diferentes (e.g., canteras, barrancos). Además, los hábitats que conforman los roquedales suelen ser relativamente pequeños en comparación con la matriz que los rodea, lo que contribuye a la idea de que los roquedales son sitios comparativamente poco relevantes (Hunter Jr. et al. 2017). Este es uno de los motivos por los cuales se los suele excluir de la mayoría de las prácticas de conservación, a menudo diseñadas para ambientes más extensos (Bauer et al. 2017; Hunter Jr. et al. 2017).

Si bien las acciones específicas para la protección de roquedales dependerán de los diferentes paisajes y ecosistemas (Fitzsimons and Michael 2017), es importante que en todas ellas se busque conservar los procesos naturales (Hjort et al. 2015) y reducir el impacto de las actividades humanas. En la Argentina, la mayor parte de los trabajos fue realizada en la región de la estepa patagónica, donde las diversas especies aprovechan la heterogeneidad que los afloramientos otorgan al paisaje plano. La Argentina posee otros numerosos paisajes con roquedales de gran valor ecológico y turístico que podrían encontrarse sometidos a disturbios; sin embargo, aún es necesario realizar estudios que analicen el impacto antrópico al que se encuentran sometidos en relación con el rol que cumplen. Esta información es fundamental para que los tomadores de decisiones puedan llevar adelante acciones efectivas que permitan conservar el patrimonio natural del país. La presencia y la representatividad de los roquedales en los sistemas de áreas protegidas y el conocimiento y la valoración por parte de científicos, manejadores de recursos y público en general, permitirá conservar los procesos ecológicos asociados a estos ambientes inertes, pero de gran valor para la conservación de la biodiversidad.

Agradecimientos. Agradecemos los comentarios recibidos por J. Guido (y su ayuda con los mapas), A. Di Virgilio, N. Rebolo, L. Zamora, P. Plaza, P. Alarcón y F. Ballejo, dos revisores anónimos y el editor asociado.

\section{REFERENCIAS}

Abdala, C. S., A. S. Quinteros, and R. E. Espinoza. 2008. Two new species of Liolaemus (Iguania: Liolaemidae) from the Puna of northwestern Argentina. Herpetologica 64:458-471.

Anchorena, J., and A. Cingolani. 2002. Identifying habitat types in a disturbed area of the forest-steppe ecotone of Patagonia. Plant Ecology 158:97-112.

Andino, N., C. E. Borghi, and S. M. Giannoni. 2016. Characterization and selection of microhabitat of Microcavia australis (Rodentia: Caviidae): first data in a rocky habitat in the hyperarid Monte Desert of Argentina. Mammalia 80:71-81.

Ávila, L. J., C. H. F. Pérez, C. D. Medina, J. W. Sites Jr., and M. Morando. 2012. A new species of lizard of the Liolaemus elongatus clade (Reptilia: Iguania: Liolaemini) from Curi Leuvu River Valley, northern Patagonia, Neuquén, Argentina. Zootaxa 3325:37-52.

Bauer, D. M., K. P. Bell, E. J. Nelson, and A. J. Calhoun. 2017. Managing small natural features: A synthesis of economic issues and emergent opportunities. Biological Conservation 211:80-87.

Braun, J. K., and M. A. Mares. 1996. Unusual morphological and behavioral traits in Abrocoma (Rodentia: Abrocomidae) from Argentina. Journal of Mammalogy 77:891-897.

Brightsmith, D. J., A. Stronza, and K. Holle. 2008. Ecotourism, conservation biology, and volunteer tourism: A mutually beneficial triumvirate. Biological Conservation 141:2832-2842.

Burke, A., K. J. Esler, E. Pienaar, and P. Barnard. 2003. Species richness and floristic relationships between mesas and their surroundings in southern African Nama Karoo. Diversity and Distributions 9:43-53.

Burnett, M. R., P. V. August, J. H. Brown, and K. T. Killingbeck. 1998. The Influence of Geomorphological Heterogeneity on Biodiversity I. A Patch-Scale Perspective. Conservation Biology 12:363-370.

Cabezas-Cartes, F., E. L. Kubisch, and N. R. Ibargüengoytía. 2014. Consequences of volcanic ash deposition on the locomotor performance of the Phymaturus spectabilis lizard from Patagonia, Argentina. Journal of Experimental Zoology Part A: Ecological Genetics and Physiology 321:164-172.

Cabezas-Cartes, F., J. Boretto, J. C. Acosta, G. Jahn, G. Blanco, A. Laspiur, and N. R. Ibargüengoytía. 2010. Reproductive 
biology of Phymaturus cf. palluma: a vulnerable lizard from the highlands of the Andes, San Juan, Argentina. Herpetological Conservation and Biology 5:430-440.

Campos, V. E., N. Andino, F. M. Cappa, M. L. Reus, and S. M. Giannoni. 2013. Microhabitat selection by Octomys mimax (Rodentia: Octodontidae) in the Monte Desert is affected by attributes and thermal properties of crevices. Revista Chilena de Historia Natural 86:315-324.

Cantero, J. J., J. A. Sfragulla, C. Núñez, J. Mulko, A. A. Bonalumi, A. Amuchastegui, G. E. Barboza, F. Chiarini, and L. Ariza Espinar. 2014. Vegetación de afloramientos carbonáticos de montañas del centro de Argentina. Boletín de la Sociedad Argentina de Botánica 49:559-580.

Cantero, J. J., J. Mulko, C. Núñez, S. R. Zeballos, J. A. Sfragulla, A. Amuchastegui, G. E. Barboza, F. Chiarini, L. Ariza Espinar, and A. A. Bonalumi. 2017. Heterogeneidad de la vegetación en ambientes basálticos del centro de Argentina. Boletín de la Sociedad Argentina de Botánica 52:153-183.

Carretero, E. M., A. D. Dalmasso, J. Márquez, and M. Martinelli. 2010. Plant communities and phytogeographical units from NW San Juan Province (High Central Andes of Argentina). Candollea 65:69-93.

Castro, S. A., A. Laspiur, and J. C. Acosta. 2013. Variación anual e intrapoblacional de la dieta de Phymaturus cf. palluma (Iguania: Liolaemidae) de los Andes centrales en Argentina. Revista Mexicana de Biodiversidad 84:1258-1265.

Ciciarelli, M. M. 2015. Canna tandilensis Ciciar. (Cannaceae-Zingiberales), una especie nueva para Argentina. Botanica Complutensis 39:87-96.

Clements, R., N. S. Sodhi, M. Schilthuizen, and P. K. Ng. 2006. Limestone karsts of Southeast Asia: imperiled arks of biodiversity. AIBS Bulletin 56:733-742.

Cole, D. N. 1993. Minimizing conflict between recreation and nature conservation. Pp. 105-122 in D. S. Smith and P. C. Hellmund (eds.). Ecology of greenways: design and function of linear conservation areas. University of Minnesota Press, Minneapolis, Minnesota, USA.

Corbalán, V., and G. Debandi. 2013. Basking behaviour in two sympatric herbivorous lizards (Liolaemidae: Phymaturus) from the Payunia volcanic region of Argentina. Journal of Natural History 47:1365-1378.

Córdoba, M. A., J. C. Acosta, H. J. Villavicencio, and V. Astudillo. 2015. Análisis trófico de Phymaturus punae (Iguania: Liolaemidae): variación estacional y sexual en la región más austral de la puna argentina. Revista Mexicana de Biodiversidad 86:1004-1013.

Czechowski, W. 2004. Scarcity of sites suitable for nesting promotes plesiobiosis in ants (Hymenoptera: Formicidae). Entomologica Fennica 15:211-218.

Debandi, G., V. Corbalan, J. A. Scolaro, and S. A. Roig-Juñent. 2012. Predicting the environmental niche of the genus Phymaturus: Are palluma and patagonicus groups ecologically differentiated? Austral Ecology 37:392-400.

Díaz, S., U. Pascual, M. Stenseke, B. Martín-López, R. T. Watson, Z. Molnár, R. Hill, K. M. Chan, I. A. Baste, and K. A. Brauman. 2018. Assessing nature's contributions to people. Science 359:270-272.

Donázar, J. A., A. Travaini, A. Rodríguez, O. Ceballos, and F. Hiraldo. 1996. Nesting association of raptors and buffnecked ibis in the Argentinean Patagonia. Colonial Waterbirds 19:111-115.

Donázar, J. A., and J. E. Feijóo. 2002. Social structure of Andean Condor roosts: influence of sex, age, and season. The Condor 104:832-837.

Dudinszky, N., and L. Ghermandi. 2013. Fire as a stimulant of shrub recruitment in northwestern Patagonian (Argentina) grasslands. Ecological Research 28:981-990.

Fandiño, B., J. M. Fernández, M. L. Thomann, R. Cajade, and A. B. Hernando. 2017. Comunidades de aves de bosques y pastizales en los afloramientos rocosos aislados del Paraje Tres Cerros, Corrientes, Argentina. Revista de Biologia Tropical 65:535-550.

Ferretti, N., G. Pompozzi, and P. Cardoso. 2017. Species conservation profile of the rare and endemic trapdoor spider Calathotarsus simoni (Araneae, Migidae) from Central Argentina. Biodiversity Data Journal 5:e14790.

Fitzsimons, J. A., and D. R. Michael. 2017. Rocky outcrops: a hard road in the conservation of critical habitats. Biological Conservation 211:36-44.

Frere, E., and P. Gandini. 2001. Aspects of the breeding biology of the Red-legged Cormorant Phalacrocorax gaimardi on the Atlantic coast of South America. Marine Ornithology 29:67-70.

Funes, G., and M. Cabido. 2008. Relaciones florísticas y características regenerativas en Apurimacia dolichocarpa (Fabaceae), especie endémica del centro de Argentina. Phytocoenologia 38:107-115.

Galende, G. I., and A. Trejo. 2003. Depredación del águila mora (Geranoaetus melanoleucus) y el búho (Bubo magellanicus) sobre el chinchillón (Lagidium viscacia) en dos colonias del noroeste de Patagonia, Argentina. Mastozoología Neotropical 10:143-147.

Galende, G. I., and D. Grigera. 1998. Relaciones alimentarias de Lagidium viscacia (Rodentia, Chinchillidae) con herbívoros introducidos en el parque nacional Nahuel Huapi, Argentina. Mastozoología Neotropical SAREM 5:123-128.

Galende, G. I., and E. Raffaele. 2008. Space use of a non-native species, the European hare (Lepus europaeus), in habitats of the southern vizcacha (Lagidium viscacia) in Northwestern Patagonia, Argentina. European Journal of Wildlife Research 54:299-304.

Galende, G. I., and E. Raffaele. 2012. Diet selection of the southern vizcacha (Lagidium viscacia): a rock specialist in north western Patagonian steppe, Argentina. Acta Theriologica 57:333-341.

Gandullo, R., and A. M. Faggi. 2006. La vegetación rupícola del Parque Provincial Copahue: Neuquén, Argentina. Kurtziana 32:13-24. 
Herrera, L., M. Sabatino, A. Gastón, and S. Saura. 2017. Grassland connectivity explains entomophilous plant species assemblages in an agricultural landscape of the Pampa Region, Argentina. Austral Ecology 42:486-496.

Hjort, J., J. E. Gordon, M. Gray, and M. L. Hunter. 2015. Why geodiversity matters in valuing nature's stage. Conservation Biology 29:630-639.

Holzschuh, A. 2016. Does rock climbing threaten cliff biodiversity? A critical review. Biological Conservation 204: 153-162.

Hunter Jr., M. L., V. Acuña, D. M. Bauer, K. P. Bell, A. J. Calhoun, M. R. Felipe-Lucia, J. A. Fitzsimons, E. González, M. Kinnison, and D. Lindenmayer. 2017. Conserving small natural features with large ecological roles: a synthetic overview. Biological Conservation 211:88-95.

IUCN 2018. The IUCN Red List of Threatened Species. Versión 2019-1. URL: www.iucnredlist.org.

Keller, H. A., and G. C. Giberti. 2011. Primer registro para la flora argentina de Ilex affinis (Aquifoliaceae), sustituto de la" yerba mate". Boletín de la Sociedad Argentina de Botánica 46:187-194.

Kitzberger, T., T. T. Veblen, and R. Villalba. 1997. Climatic influences on fire regimes along a rain forest-to-xeric woodland gradient in northern Patagonia, Argentina. Journal of Biogeography 24:35-47.

Köhler, J., and W. Böhme. 1996. Anuran amphibians from the region of Pre-Cambrian rock outcrops (inselbergs) in northeastern Bolivia, with a note on the gender of Scinax Wagler, 1830 (Hylidae). Revue Française d'Aquariologie Herpétologie 23:133-140.

Kristensen, M. J., and J. L. Frangi. 2015. Chasmophytic vegetation and mesoclimates of rock outcrops in Ventania (Buenos Aires, Argentina). Boletín de la Sociedad Argentina de Botánica 50:35-46.

Lambertucci, S. A., and A. Ruggiero. 2013. Cliffs used as communal roosts by Andean Condors protect the birds from weather and predators. PloS one 8:e67304.

Lambertucci, S. A., and A. Ruggiero. 2016. Cliff outcrops used as condor communal roosts are local hotspots of occupancy and intense use by other bird species. Biological Conservation 200:8-16.

Lambertucci, S. A., and K. L. Speziale. 2009. Some possible anthropogenic threats to breeding Andean condors (Vultur gryphus). Journal of Raptor Research 43:245-249.

Lambertucci, S. A., and O. A. Mastrantuoni. 2008. Breeding behavior of a pair of free-living Andean Condors. Journal of Field Ornithology 79:147-151.

Lambertucci, S. A., N. Luis Jácome, and A. Trejo. 2008. Use of communal roosts by Andean Condors in northwest Patagonia, Argentina. Journal of Field Ornithology 79:138-146.

Landesmann, J. B., J. H. Gowda, L. A. Garibaldi, and T. Kitzberger. 2015. Survival, growth and vulnerability to drought in fire refuges: implications for the persistence of a fire-sensitive conifer in northern Patagonia. Oecologia 179:11111122.

Larson, D. W., U. Matthes, and P. E. Kelly. 2000. Cliff ecology: pattern and process in cliff ecosystems. Cambridge University Press, Cambridge, UK.

Lewis, J., and B. Lewis. 2017. Rock art and mining violence on the Australian Burrup Peninsula: language wars, economy and culture. Perspectives in Ecology and Conservation 15:179-186.

Mares, M. A. 1997. The geobiological interface: Granitic outcrops as a selective force in mammalian evolution. Journal of the Royal Society of Western Australia 80:131-139.

Mares, M. A., and T. E. Lacher. 1987. Ecological, morphological, and behavioral convergence in rock-dwelling mammals. Pp. 307-348 in Current mammalogy. Springer, Boston, MA, USA.

Menghi, M., M. Cabido, B. Peco, and F. D. Pineda. 1989. Grassland heterogeneity in relation to lithology and geomorphology in the Córdoba Mountains, Argentina. Vegetatio 84:133-142.

Michael, D. R., and D. Lindenmayer. 2012. Vegetation structure and floristics of granite landforms in the South-west Slopes of New South Wales. Cunninghamia 12:309-323.

Michael, D. R., D. B. Lindenmayer, and R. B. Cunningham. 2010. Managing rock outcrops to improve biodiversity conservation in Australian agricultural landscapes. Ecological Management and Restoration 11:43-50.

Michael, D. R., R. B. Cunningham, and D. B. Lindenmayer. 2008. A forgotten habitat? Granite inselbergs conserve reptile diversity in fragmented agricultural landscapes. Journal of Applied Ecology 45:1742-1752.

Milchunas, D. G., and I. Noy-Meir. 2002. Grazing refuges, external avoidance of herbivory and plant diversity. Oikos 99:113-130.

Moncrieff, D. 2000. Managing tourism and recreation on Wheatbelt granite outcrops. Journal of the Royal Society of Western Australia 83:187-196.

Morrison, D. A., G. J. Cary, S. M. Pengelly, D. G. Ross, B. J. Mullins, C. R. Thomas, and T. S. Anderson. 1995. Effects of fire frequency on plant species composition of sandstone communities in the Sydney region: Inter-fire interval and time-since-fire. Austral Ecology 20:239-247.

Oddi, F. J., N. Dudinszky, and L. Ghermandi. 2010. Spatial dynamics of Fabiana imbricata shrublands in northwestern Patagonia in relation to natural fires. Natural Hazards and Earth System Sciences 10:957-966.

Paukov, A. G. 2009. The lichen flora of serpentine outcrops in the Middle Urals of Russia. Northeastern Naturalist 16:341-350.

Pia, M. V., D. Renison, A. Mangeaud, C. De Angelo, and J. G. Haro. 2013. Occurrence of top carnivores in relation to land protection status, human settlements and rock outcrops in the high mountains of central Argentina. Journal of Arid Environments 91:31-37. 
Pike, D. A., B. M. Croak, J. K. Webb, and R. Shine. 2010. Subtle-but easily reversible-anthropogenic disturbance seriously degrades habitat quality for rock-dwelling reptiles. Animal Conservation 13:411-418.

Porembski, S., and W. Barthlott. 2000. Inselbergs: biotic diversity of isolated rock outcrops in tropical and temperate regions. Springer Science and Business Media, Berlin, Germany.

Puig, S., F. Videla, M. Cona, S. Monge, and U. Roig. 1998. Diet of the Mountain vizcacha (Logidium viscacia Molina, 1782) and food availability in northern Patagonia, Argentina. Zeitschrift fur Saugetierkunde 63:228-238.

Punta, G., P. Yorio, J. Saravia, and P. García Borboroglu. 2003. Breeding habitat requirements of the Imperial Cormorant and Rock Shag in central Patagonia, Argentina. Waterbirds 26:176-183.

Quinteros, A. S., C. H. F. Pérez, N. Pelegrín, M. Morando, R. Montero, A. Laspiur, F. Kacoliris, N. R. Ibarguengoytía, A. Hernando, and V. Corbalán. 2012. Categorización del estado de conservación de las lagartijas y anfisbenas de la República Argentina. Cuadernos de Herpetología 26:215-248.

Renison, D., A. M. Cingolani, and R. Suárez. 2002. Effects of fire on a Polylepis australis (Rosaceae) woodland in the mountains of Cordoba, Argentina. Revista Chilena de Historia Natural 75:719-727.

Roesler, I., S. Imberti, H. E. Casañas, P. M. Hernández, J. M. Klavins, and L. G. Pagano. 2014. Noteworthy records and natural history comments on rare and threatened bird species from Santa Cruz province, Patagonia, Argentina. Revista Brasileira de Ornitologia-Brazilian Journal of Ornithology 22:189-200.

Roitman, G. G. 1999. Pollination biology of Grindelia covasii (Asteraceae), a potential crop for arid lands. Journal of Arid Environments 43:103-110.

Rosciano, N. G., W. S. Svagelj, and A. Raya Rey. 2013. Effect of anthropic activity on the Imperial Cormorants and Rock Shags colonies in the Beagle Channel, Tierra del Fuego. Revista de Biología Marina y Oceanografía 48:165-176.

Rossi, A. M., and R. E. Webb. 2007. The consequences of allowing unrestricted tourist access at an Aboriginal site in a fragile environment: The erosive effect of trampling. Conservation and Management of Archaeological Sites 9: 219-236.

Scolaro, J. A., and N. R. Ibargüengoytía. 2008. A new fragment for the understanding of the puzzling evolutive process of the Phymaturus genus: a new species of the patagonicus group from Patagonia, Argentina (Reptilia: Iguania: Liolaemidae). Zootaxa 1939:38-50.

Sobrero, R., V. E. Campos, S. M. Giannoni, and L. A. Ebensperger. 2010. Octomys mimax (Rodentia: Octodontidae). Mammalian Species 42:49-57.

Søchting, U., M. Z. Søgaard, A. Ulf, A. Elvebakk, and L. G. Sancho. 2014. Catenarina (Teloschistaceae, Ascomycota), a new Southern Hemisphere genus with 7-chlorocatenarin. The Lichenologist 46:175-187.

Souto, C. P., K. Heinemann, T. Kitzberger, A. C. Newton, and A. C. Premoli. 2012. Genetic diversity and structure in Austrocedrus chilensis populations: Implications for dryland forest restoration. Restoration Ecology 20:568-575.

Speziale, K. L., S. A. Lambertucci, M. Carrete, and J. L. Tella. 2012. Dealing with non-native species: what makes the difference in South America? Biological Invasions 14:1609-1621.

Speziale, K. L., and C. Ezcurra. 2012. The role of outcrops in the diversity of Patagonian vegetation: Relicts of glacial palaeofloras? Flora-Morphology, Distribution, Functional Ecology of Plants 207:141-149.

Speziale, K. L., and C. Ezcurra. 2015. Rock outcrops as potential biodiversity refugia under climate change in North Patagonia. Plant Ecology and Diversity 8:353-361.

Taraborelli, P. A., P. L. Sassi, M. A. Dacar, P. Moreno, and R. A. Ojeda. 2015. Abrocoma schistacea (Rodentia: Abrocomidae). Mammalian Species 47:45-50.

Taraborelli, P., and P. Moreno. 2009. Comparing composition of social groups, mating system and social behaviour in two populations of Microcavia australis. Mammalian Biology-Zeitschrift für Säugetierkunde 74:15-24.

Taraborelli, P., P. Moreno, P. Sassi, M. A. Dacar, and R. Ojeda. 2011. New eco-morphological-behavioural approach of the chinchilla rats in the pre-Andean foothills of the Monte Desert (Argentina). Journal of natural history 45:1745-1758.

Tassara, D. A., and M. M. Cenizo. 2014. El patrimonio paleontológico en el sector costero al NE de Mar del Plata (Provincia de Buenos Aires, Argentina): Estado del conocimiento, vulnerabilidad y propuestas para su conservación. Revista del Museo Argentino de Ciencias Naturales 16:165-183.

Tscharntke, T., A. M. Klein, A. Kruess, I. Steffan-Dewenter, and C. Thies. 2005. Landscape perspectives on agricultural intensification and biodiversity-ecosystem service management. Ecology Letters 8:857-874.

Vischi, N., E. Natale, and C. Villamil. 2004. Six endemic plant species from central Argentina: an evaluation of their conservation status. Biodiversity and Conservation 13:997-1008.

Walker, R. S., A. J. Novaro, and L. C. Branch. 2003. Effects of patch attributes, barriers, and distance between patches on the distribution of a rock-dwelling rodent (Lagidium viscacia). Landscape Ecology 18:185-192.

Walker, R. S., G. Ackermann, J. Schachter-Broide, V. Pancotto, and A. J. Novaro. 2000. Habitat use by mountain vizcachas (Lagidium viscacia Molina, 1782) in the Patagonian steppe. Mammalian Biology-Zeitschrift fur Saugetierkunde 65: 293-300.

Ward, P., and A. Zahavi. 1973. The importance of certain assemblages of birds as "information-centres" for foodfinding. Ibis 115:517-534. 